arxiv:1012.3982, AEI-2010-175

CERN-PH-TH/2010-306, HU-EP-10/87

HU-MATH-2010-22, kcl-mth-10-10

UMTG-270, UUITP-41/10

\title{
Review of AdS/CFT Integrability:
} An Overview

\author{
Niklas Beisert ${ }^{\dagger} 1$, Changrim Ahn ${ }^{2}$, Luis F. Alday ${ }^{34}$, Zoltán Bajnok ${ }^{5}$, \\ James M. Drummond ${ }^{67}$, Lisa Freyhult ${ }^{8}$, Nikolay Gromovi10, \\ Romuald A. Janik ${ }^{11}$, Vladimir Kazakov ${ }^{12}{ }^{13}$, Thomas Klose ${ }^{814}$, \\ Gregory P. Korchemsky ${ }^{15}$, Charlotte Kristuansen ${ }^{16}$, Marc Magro ${ }^{171}$, \\ Tristan Mcloughlin ${ }^{1}$, Joseph A. Minahan ${ }^{8}$, Rafael I. Nepomechie ${ }^{18}$, \\ AdAm Rej ${ }^{19}$, Radu Roiban ${ }^{20}$, SAKura SchäfER-NAMEKi ${ }^{921}$, \\ Christoph Sieg ${ }^{2223}$, Matthias Staudacher ${ }^{221}$, Alessandro Torrielli ${ }^{2425}$, \\ Arkady A. Tseytlin ${ }^{19}$, Pedro Vieira ${ }^{26}$, Dmytro Volin ${ }^{20}$ And \\ Konstantinos Zoubos ${ }^{16}$ \\ ${ }^{\dagger}$ corresponding author, e-mail address: nbeisert@aei.mpg.de
}

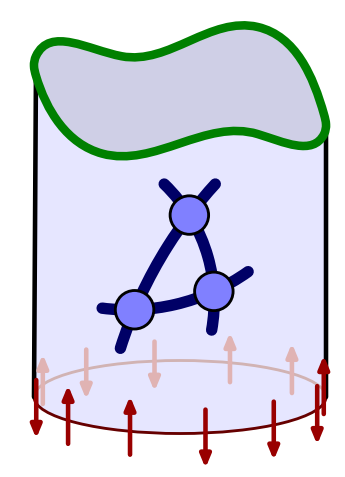

Abstract: This is the introductory chapter of a review collection on integrability in the context of the AdS/CFT correspondence. In the collection we present an overview of the achievements and the status of this subject as of the year 2010. 


\section{Addresses:}

1 Max-Planck-Institut für Gravitationsphysik, Albert-Einstein-Institut Am Mühlenberg 1, 14476 Potsdam, Germany

2 Department of Physics and Institute for the Early Universe Ewha Womans University, Seoul 120-750, South Korea

3 Mathematical Institute, University of Oxford 24-29 St. Giles', Oxford OX1 3LB, U.K.

4 School of Natural Sciences, Institute for Advanced Study, Princeton, NJ 08540, USA

5 Theoretical Physics Research Group of the Hungarian Academy of Sciences 1117 Pázmány s. 1/A, Budapest, Hungary

6 PH-TH Division, CERN, Geneva, Switzerland

7 LAPTH, Université de Savoie, CNRS B.P. 110, 74941 Annecy-le-Vieux Cedex, France

8 Department of Physics and Astronomy, Division for Theoretical Physics Uppsala University, Box 516, SE-751 08 Uppsala, Sweden

9 Department of Mathematics, King's College, The Strand, London, WC2R 2LS, U.K.

10 PNPI, Gatchina, Leningrad District, 188300 St. Petersburg, Russia

11 Institute of Physics, Jagiellonian University ul. Reymonta 4, 30-059 Kraków, Poland

12 LPT, Ecole Normale Superiéure, 24 rue Lhomond, 75231 Paris Cedex 05, France

13 Université Pierre et Marie Curie (Paris-VI), 4 Place Jussieu, 75252 Paris Cedex 05, France

14 Princeton Center for Theoretical Science Princeton University, Princeton, NJ 08544, USA

15 Institut de Physique Théorique, CEA Saclay 91191 Gif-sur-Yvette Cedex, France

16 The Niels Bohr Institute, Blegdamsvej 17, 2100 Copenhagen, Denmark

17 Université de Lyon, Laboratoire de Physique, ENS Lyon et CNRS UMR 5672, 46 allée d'Italie, 69364 Lyon CEDEX 07, France

18 Physics Department, P.O. Box 248046, University of Miami Coral Gables, FL 33124, USA

19 Blackett Laboratory, Imperial College London, SW7 2AZ, U.K.

20 Department of Physics, The Pennsylvania State University University Park, PA 16802, USA

21 Kavli Institute for Theoretical Physics, University of California, Santa Barbara, CA 93106, USA

22 Institut für Mathematik und Institut für Physik, Humboldt-Universität zu Berlin Johann von Neumann-Haus, Rudower Chaussee 25, 12489 Berlin, Germany

23 Niels Bohr International Academy, Niels Bohr Institute Blegdamsvej 17, 2100 Copenhagen, Denmark

24 Department of Mathematics, University of York Heslington, York, YO10 5DD, U.K.

25 Institute for Theoretical Physics, Utrecht University Leuvenlaan 4, 3584 CE Utrecht, The Netherlands

26 Perimeter Institute for Theoretical Physics, Waterloo, Ontario N2L 2Y5, Canada 


\section{Preface}

Since late 2002 tremendous and rapid progress has been made in exploring planar $\mathcal{N}=$ 4 super Yang-Mills theory and free IIB superstrings on the $A d S_{5} \times S^{5}$ background. These two models are claimed to be exactly dual by the AdS/CFT correspondence, and the novel results give full support to the duality. The key to this progress lies in the integrability of the free/planar sector of the AdS/CFT pair of models.

Many reviews of integrability in the context of the AdS/CFT correspondence are available in the literature. They cover selected branches of the subject which have appeared over the years. Still it becomes increasingly difficult to maintain an overview of the entire subject, even for experts. Already for several years there has been a clear demand for an up-to-date review to present a global view and summary of the subject, its motivation, techniques, results and implications.

Such a review appears to be a daunting task: With around 8 years of development and perhaps up to 1000 scientific articles written, the preparation would represent a major burden on the prospective authors. Therefore, our idea was to prepare a coordinated review collection to fill the gap of a missing global review for AdS/CFT integrability. Coordination consisted in carefully splitting up the subject into a number of coherent topics. These cover most aspects of the subject without overlapping too much. Each topic is reviewed by someone who has made important contributions to it. The collection is aimed at beginning students and at scientists working on different subjects, but also at experts who would like to (re)acquire an overview. Special care was taken to keep the chapters brief (around 20 pages), focused and self-contained in order to enable the interested reader to absorb a selected topic in one go.

As the individual chapters will not convey an overview of the subject as a whole, the purpose of the introductory chapter is to assemble the pieces of the puzzle into a bigger picture. It consists of two parts: The first part is a general review of AdS/CFT integrability. It concentrates on setting the scene, outlining the achievements and putting them into context. It tries to provide a qualitative understanding of what integrability is good for and how and why it works. The second part maps out how the topics/chapters fit together and make up the subject. It also contains sketches of the contents of each chapter. This part helps the reader in identifying the chapters (s)he is most interested in.

There are reasons for and against combining all the contributions into one article or book. Practical issues however make it advisable to have the chapters appear as autonomous review articles. After all, they are the works of individuals. They are merely tied together by the introductory chapter on which all the contributors have signed as coauthors. If you wish to refer to this review on AdS/CFT integrability as a whole, we suggest that you cite (only) the introductory chapter:

N. Beisert et al.,

"Review of AdS/CFT Integrability: An Overview",

Lett. Math. Phys. 99, 3 (2012), arXiv:1012.3982.

If your work refers to a particular topic of the review, we encourage you to cite the corresponding specialised chapter(s) (instead/in addition), e.g. 


\section{J. A. Minahan,}

"Review of AdS/CFT Integrability, Chapter I.1: Spin Chains in $\mathcal{N}=4$ SYM", Lett. Math. Phys. 99, 33 (2012), arXiv:1012.3983.

Finally, I would like to thank my coauthors for their collaboration on this project. In particular, I am grateful to Pedro Vieira who set up a website for internal discussions which facilitated the coordination greatly: Drafts and outlines of the chapters were uploaded to this forum. Here, the contributors to the collection gave helpful comments and suggestions on the other chapters. It is fair to say that the forum improved the quality and completeness of the articles and how they fit together before they first appeared in public. Also managing the final production stage would not have been nearly as efficient without it. Thanks for all your help and prompt availability during the last week!

Niklas Beisert Potsdam, December 2010 


\section{Introduction}

An old dream of Quantum Field Theory (QFT) is to derive a quantitative description of the mass spectrum of hadronic particles and their excitations. Ideally, one would be able to express the masses of particles such as protons and neutrons as functions of the parameters of the theory

$$
m_{\mathrm{p}}=f_{1}\left(\alpha_{\mathrm{s}}, \alpha, \mu_{\mathrm{reg}}, \ldots\right), \quad m_{\mathrm{n}}=f_{2}\left(\alpha_{\mathrm{s}}, \alpha, \mu_{\mathrm{reg}}, \ldots\right), \quad \ldots
$$

They might be combinations of elementary functions, solutions to differential or integral equations or something that can be evaluated effortlessly on a present-day computer. For the energy levels of the hydrogen atom analogous functions are known and they work to a high accuracy. However, it has become clear that an elementary analytical understanding of the hadron spectrum will remain a dream. There are many reasons why this is more than can be expected; just to mention a few: At low energies, the coupling constant $\alpha_{\mathrm{s}}$ is too large for meaningful approximations. In particular, non-perturbative contributions dominate such that the standard loop expansion simply does not apply. Self-interactions of the chromodynamic field lead to a non-linear and highly complex problem. Clearly, confinement obscures the nature of fundamental particles in Quantum Chromodynamics (QCD) at low energies. Of course there are non-perturbative methods to arrive at reasonable approximations for the spectrum, but these are typically based on effective field theory or elaborate numerical simulations instead of elementary analytical QCD.

Spectrum of Scaling Dimensions. We shall use the above hadronic spectrum as an analog to explain the progress in applying methods of integrability to the spectrum of planar $\mathcal{N}=4$ super Yang-Mills (SYM) theory. ${ }^{1}$ The analogy does not go all the way, certainly not at a technical level, but it is still useful for a qualitative understanding of the achievements.

First of all, $\mathcal{N}=4 \mathrm{SYM}$ is a cousin of QCD and of the Standard Model of particle physics. It is based on the same types of fundamental particles and interactions - it is a renormalisable gauge field theory on four-dimensional Minkowski space - but the details of the models are different. Importantly, $\mathcal{N}=4 \mathrm{SYM}$ has a much richer set of symmetries: supersymmetry and conformal symmetry. In particular, the latter implies that there are no massive particles whose spectrum we might wish to compute. Nevertheless, composite particles and their mass spectrum have an analogue in conformal field theories: These are called local operators. They are composed from the fundamental fields, all residing at a common point in spacetime. As in QCD, the colour charges are balanced out making the composites gauge-invariant objects. Last but not least, there is a characteristic quantity to replace the mass, the so-called scaling dimension. Classically, it equals the sum of the constituent dimensions, and, like the mass, it does receive quantum corrections (the so-called anomalous dimensions) from interactions between the constituents.

\footnotetext{
${ }^{1}$ Please note that, here and below, references to the original literature can be found in the chapters of this review collection where the underlying models are introduced.
} 
In the planar $\mathcal{N}=4 \mathrm{SYM}$ model and for scaling dimensions of local operators the particle physicist's dream is coming true. We know how to express the scaling dimension $D_{O}$ of some local operator $O$ as a function of the coupling constant $\lambda$

$$
D_{O}=f(\lambda)
$$

In general this function is given as the solution of a set of integral equations. ${ }^{2}$ What is more, in particular cases the equations have been solved numerically for a wide range of $\lambda$ 's! These equations follow from the so-called Thermodynamic Bethe Ansatz (TBA) or related techniques (Y-system). In a certain limit, the equations simplify to a set of algebraic equations, the so-called asymptotic Bethe equations. It is also becoming clear that not only the spectrum, but many other observables can be determined in this way. Thus it appears that planar $\mathcal{N}=4 \mathrm{SYM}$ can be solved exactly.

Integrability. With the new methods at hand we can now compute observables which were previously inaccessible by all practical means. By studying the observables and the solution, we hope to get novel insights, not only into this particular model, but also into quantum gauge field theory in general. What is it that makes planar $\mathcal{N}=4$ SYM calculable and other models not? Is its behaviour generic or very special? Can we for instance use the solution as a starting point or first approximation for other models? On the one hand one may view $\mathcal{N}=4 \mathrm{SYM}$ as a very special QFT. On the other hand, any other four-dimensional gauge theory can be viewed as $\mathcal{N}=4 \mathrm{SYM}$ with some particles and interactions added or removed: For instance, several quantities show a universal behaviour throughout the class of four-dimensional gauge theories (e.g. highest "transcendentality" part, tree-level gluon scattering). Moreover this behaviour is dictated by $\mathcal{N}=4 \mathrm{SYM}$ acting as a representative model. Thus, indeed, selected results obtained in $\mathcal{N}=4 \mathrm{SYM}$ can be carried over to general gauge theories. Nevertheless it is obvious that we cannot make direct predictions along these lines for most observables, such as the hadron spectrum.

The miracle which leads to the solution of planar $\mathcal{N}=4 \mathrm{SYM}$ described above is generally called integrability. Integrability is a phenomenon which is typically confined to two-dimensional models (of Euclidean or Minkowski signature). Oddly, here it helps in solving a four-dimensional QFT.

AdS/CFT Correspondence. A more intuitive understanding of why there is integrability comes from the AdS/CFT correspondence [1], see also the reviews [2] and [3]. The latter is a duality relation between certain pairs of models. One partner is a conformal field theory, i.e. a QFT with exact conformal spacetime symmetry. The other partner is a string theory where the strings propagate on a background which contains an Anti-de-Sitter spacetime (AdS) as a factor. The boundary of an $A d S_{d+1}$ spacetime is a conformally flat $d$-dimensional spacetime on which the CFT is formulated. The AdS/CFT duality relates the string partition function with sources $\phi$ for string vertex

\footnotetext{
${ }^{2}$ As a matter of fact, the system of equations is not yet in a form which enables easy evaluation. E.g. there are infinitely many equations for infinitely many quantities. It is however common belief that one can, as in similar cases, reduce the system to a finite set of Non-Linear Integral Equations (NLIE).
} 
operators fixed to value $J$ at the boundary of $A d S_{d+1}$ to the $\mathrm{CFT}_{d}$ partition function with sources $J$ for local operators

$$
Z_{\mathrm{str}}\left[\left.\phi\right|_{\partial A d S}=J\right]=Z_{\mathrm{CFT}}[J] .
$$

More colloquially: For every string observable at the boundary of $A d S_{d+1}$ there is a corresponding observable in the $\mathrm{CFT}_{d}$ (and vice versa) whose values are expected to match. This is a remarkable statement because it relates two rather different types of models on spacetimes of different dimensionalities. From it we gain novel insights into one model through established results from the other model. For example, we can hope to learn about the long-standing problem of quantum gravity (gravity being a fundamental part of every string theory) through studying a more conventional QFT. However, this transfer of results requires a leap of faith as long as the duality lacks a formal proof.

Most attempts at testing the predictions of the AdS/CFT duality have focused on its most symmetric setting: The CFT partner is the gauge theory featured above, $\mathcal{N}=4$ SYM. The string partner is IIB superstring theory on the $A d S_{5} \times S^{5}$ background. This pair is an ideal testing ground because the large amount of supersymmetry leads to simplifications and even allows for exact statements about both models. In this context, we can also understand the miraculous appearance of integrability in planar $\mathcal{N}=4$ SYM better: By means of the AdS/CFT duality it translates to integrability of the string worldsheet model. The latter is a two-dimensional non-linear sigma model on a symmetric coset space for which integrability is a common phenomenon. Consequently, integrability has become an important tool to perform exact calculations in both models. Full agreement between both sides of the duality has been observed in all considered cases. Therefore, integrability has added substantially to the credibility of the AdS/CFT correspondence.

String/Gauge Duality. Another important aspect of the AdS/CFT duality is that in many cases it relates a string theory to a gauge theory. In fact, the insight regarding the similarities between these two types of models is as old as string theory: It is well-known that the hadron spectrum organises into so-called Regge trajectories. These represent an approximate linear relationship with universal slope between the mass squared of hadronic resonances and their spin. This is precisely what a string theory on flat space predicts, hence string theory was for some time considered a candidate model of the strong interactions. For various reasons this idea did not work out. Instead, it was found that a gauge theory, namely QCD, provides an accurate and self-consistent description of the strong interactions. Altogether it implies that string theory, under some conditions, can be a useful approximation to gauge theory phenomena. A manifestation of stringy behaviour in gauge theory is the occurrence of flux tubes of the chromodynamic field. Flux tubes form between two quarks when they are pulled apart. To some approximation they can be viewed as one-dimensional objects with constant tension, i.e. strings. The AdS/CFT correspondence goes even further. It proposes that in some cases a gauge theory is exactly dual to a string theory. By studying those cases, we hope to gain more insights into string/gauge duality in general, perhaps even for QCD.

A milestone of string/gauge duality was the discovery of the planar limit [4], see Fig. 1. This is a limit for models with gauge group $\mathrm{SU}\left(N_{\mathrm{c}}\right), \operatorname{SO}\left(N_{\mathrm{c}}\right)$ or $\operatorname{Sp}\left(N_{\mathrm{c}}\right)$. It 



Figure 1: Planar and non-planar Feynman graph (top), free and interacting string worldsheet (bottom), Feynman graph corresponding to a patch of worldsheet (right).

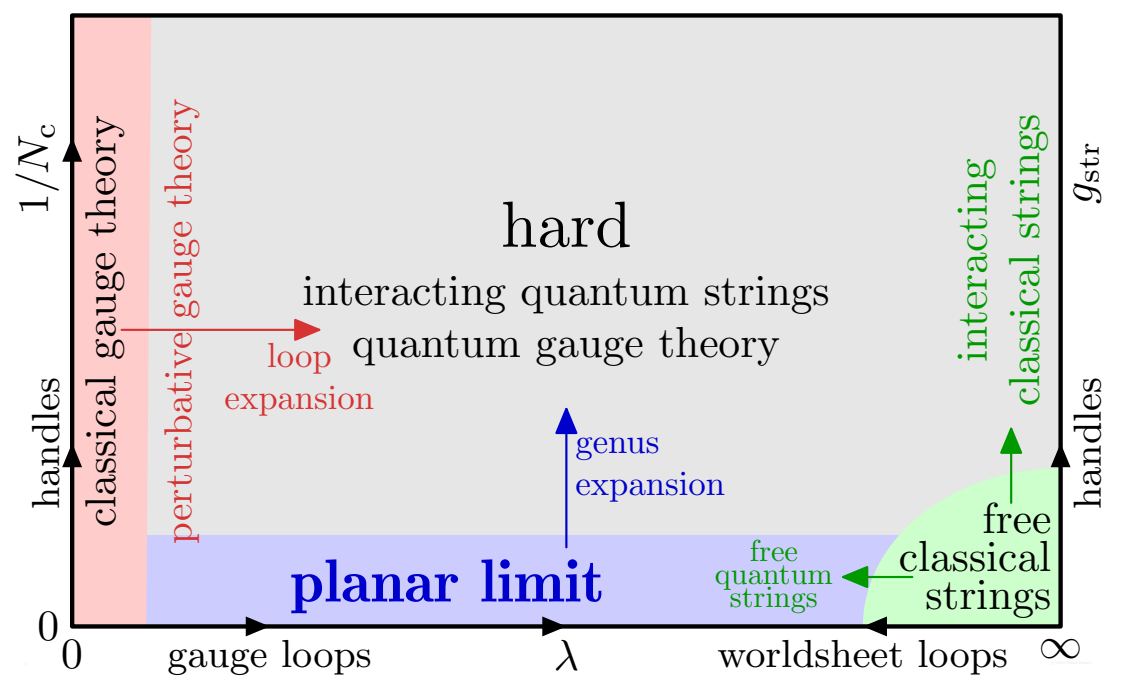

Figure 2: Map of the parameter space of $\mathcal{N}=4 \mathrm{SYM}$ or strings on $A d S_{5} \times S^{5}$.

consists in taking the rank of the group to infinity, $N_{\mathrm{c}} \rightarrow \infty$, while keeping the rescaled gauge coupling $\lambda=g_{\mathrm{YM}}^{2} N_{\mathrm{c}}$ finite. In this limit, the Feynman graphs which describe the perturbative expansion of gauge theory around $\lambda=0$ can be classified according to their genus: Graphs which can be drawn on the plane without crossing lines are called planar. The remaining graphs with crossing lines are suppressed. This substantially reduces the complexity of graphs from factorial to exponential growth, such that the radius of convergence of the perturbative series grows to a finite size. Moreover, the surface on which the Feynman graphs are drawn introduces a two-dimensional structure into gauge theory: It is analogous to the worldsheet of a string whose string coupling $g_{\mathrm{str}}$ is proportional to $1 / N_{\mathrm{c}}$. Not surprisingly, integrability is confined to this planar limit where gauge theory resembles string theory. 
Parameter Space. Let us now discuss the progress due to integrability based on a map of the parameter space of our gauge and string theory, see Fig. 2. Typically there are two relevant parameters for a gauge theory, the 't Hooft coupling $\lambda=g_{\mathrm{YM}}^{2} N_{\mathrm{c}}$ and the number of colours $N_{\mathrm{c}}$ as a measure of the rank of the gauge group. In a string theory we have the effective string tension $T=R^{2} / 2 \pi \alpha^{\prime}$ (composed from the inverse string tension $\alpha^{\prime}$ and the $A d S_{5} / S^{5}$ radius $R$ ) and the string coupling $g_{\mathrm{str}}$. The AdS/CFT correspondence relates them as follows

$$
\lambda=4 \pi^{2} T^{2}, \quad \frac{1}{N_{\mathrm{c}}}=\frac{g_{\mathrm{str}}}{4 \pi^{2} T^{2}} .
$$

The region of parameter space where $\lambda$ is small is generally called the weak coupling regime. This is where perturbative gauge theory in terms of Feynman diagrams provides reliable results. By adding more loop orders to the series expansion one can obtain more accurate estimates towards the centre of the parameter space (up to non-perturbative effects). Unfortunately, conventional methods in combination with computer algebra only allow evaluating the first few coefficients of the series in practice. Thus we cannot probe the parameter space far away from the weak coupling regime. However, $N_{\mathrm{c}}$ can be finite in practice, therefore the regime of perturbative gauge theory extends along the line $\lambda=0$.

The region around the point $\lambda=\infty, g_{\text {str }}=0$ is where perturbative string theory applies. Here, strings are weakly coupled, but the region is nevertheless called the strong coupling regime referring to the gauge theory parameter $\lambda$. String theory provides a double expansion around this point. The accuracy towards finite $\lambda$ is increased by adding quantum corrections to the worldsheet sigma model (curvature expansion, "worldsheet loops"). Finite- $g_{\text {str }}$ corrections correspond to adding handles to the string worldsheet (genus expansion, "string loops"). As before, both expansions are far from trivial, and typically only the first few coefficients can be computed in practice. Consequently, series expansions do not give reliable approximations far away from the point $\lambda=\infty, g_{\mathrm{str}}=0$.

Here we can see the weak/strong dilemma of the AdS/CFT duality, see also Fig. 3: The perturbative regimes of the two models do not overlap. On the one hand AdS/CFT provides novel insights into both models. On the other hand, we cannot really be sure of them until there is a general proof of the duality. Conventional perturbative expansions are of limited use in verifying, and tests had been possible only for a few special observables (cf. $|5|$ for example).

This is where integrability comes to help. As explained above, it provides novel computational means in planar $\mathcal{N}=4 \mathrm{SYM}$ at arbitrary coupling $\lambda$. The AdS/CFT correspondence relates this regime to free $\left(g_{\text {str }}=0\right)$ IIB superstrings on $A d S_{5} \times S^{5}$ at arbitrary tension $T$. It connects the regime of perturbative gauge theory with the regime of perturbative string theory. Integrability predicts the spectrum of planar scaling dimensions for local operators as a function of $\lambda$, cf. Fig. 3. In string theory this is dual to the energy spectrum of free string states (strings which neither break apart nor join with others). We find that integrability makes coincident predictions for both models. At weak coupling one can compare to results obtained by conventional perturbative means in gauge theory, and one finds agreement. Analogous agreement with perturbative strings 

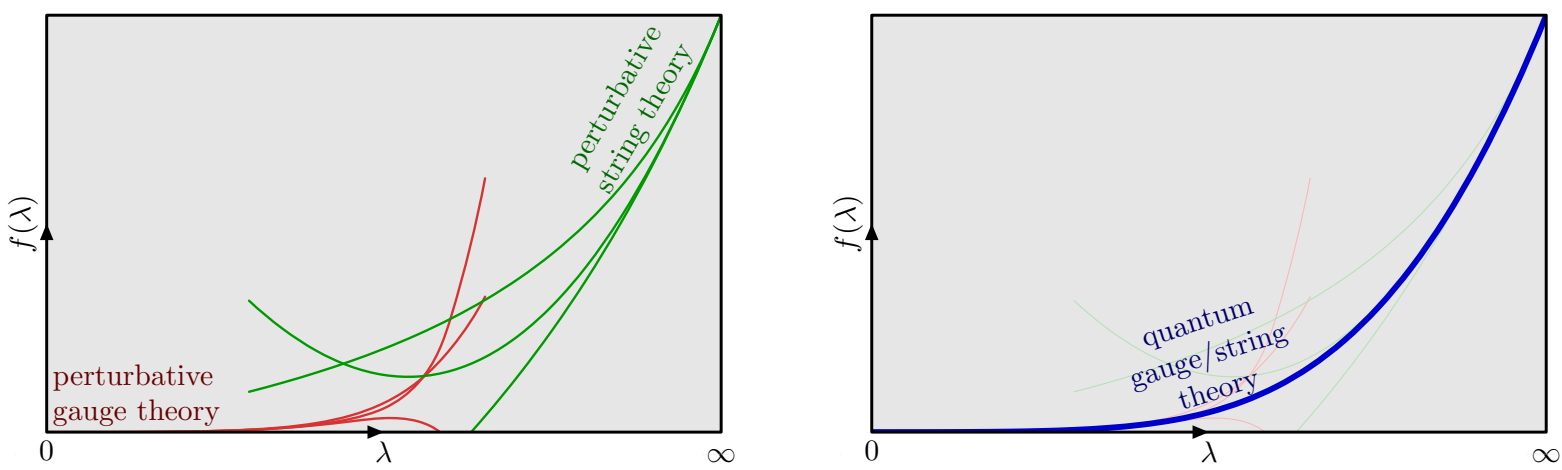

Figure 3: Weak coupling (3, 5, 7 loops) and strong coupling (0, 1, 2 loops) expansions (left) and numerically exact evaluation (right) of some interpolating function $f(\lambda)$.

is found at strong coupling. And for intermediate coupling the spectrum apparently interpolates smoothly between the two perturbative regimes.

Methods of integrability provide us with reliable data over the complete range of couplings. We can investigate in practice a gauge theory at strong coupling. There it behaves like a weakly coupled string theory. Likewise a string theory on a highly curved background (equivalent to low tension) behaves like a weakly coupled gauge theory. At intermediate coupling, the results are reminiscent of neither model or of both; this is merely a matter of taste and depends crucially on whether one's intuition is based on classical or quantum physics. In any case, integrability can give us valuable insights into a truly quantum gauge and/or string theory at intermediate coupling strength.

Solving a Theory. In conclusion, we claim that integrability solves the planar sector of a particular pair of gauge and string theories. We should be clear about the actual meaning of this statement: It certainly does not mean that the spectrum is given by a simple formula as in the case of a harmonic oscillator, the (idealised) hydrogen atom or strings in flat space (essentially a collection of harmonic oscillators) ${ }^{3}$

$$
E_{\mathrm{HO}}=\omega\left(n+\frac{1}{2}\right), \quad E_{\mathrm{hyd}}=-\frac{m_{\mathrm{e}} \alpha^{2}}{n^{2}}, \quad m_{\text {flat }}^{2}=m_{0}^{2}+\frac{1}{\alpha^{\prime}} \sum_{k=-\infty}^{\infty} n_{k}|k| .
$$

It would be too much to hope for such a simplistic behaviour in our models: For instance, the one-loop corrections to scaling dimensions are typically algebraic numbers. Therefore the best we can expect is to find a system of algebraic equations whose solutions determine the spectrum. This is what methods of integrability provide more or less directly. Integrability vastly reduces the complexity of the spectral problem by bypassing almost all steps of standard QFT methods: There we first need to compute all the entries of the matrix of scaling dimensions. Each entry requires a full-fledged computation of higher loop Feynman graphs involving sophisticated combinatorics and demanding loop integrals. The naively evaluated matrix contains infinities calling for proper regularisation

\footnotetext{
${ }^{3}$ In fact, these systems are also integrable, but of an even simpler kind.
} 


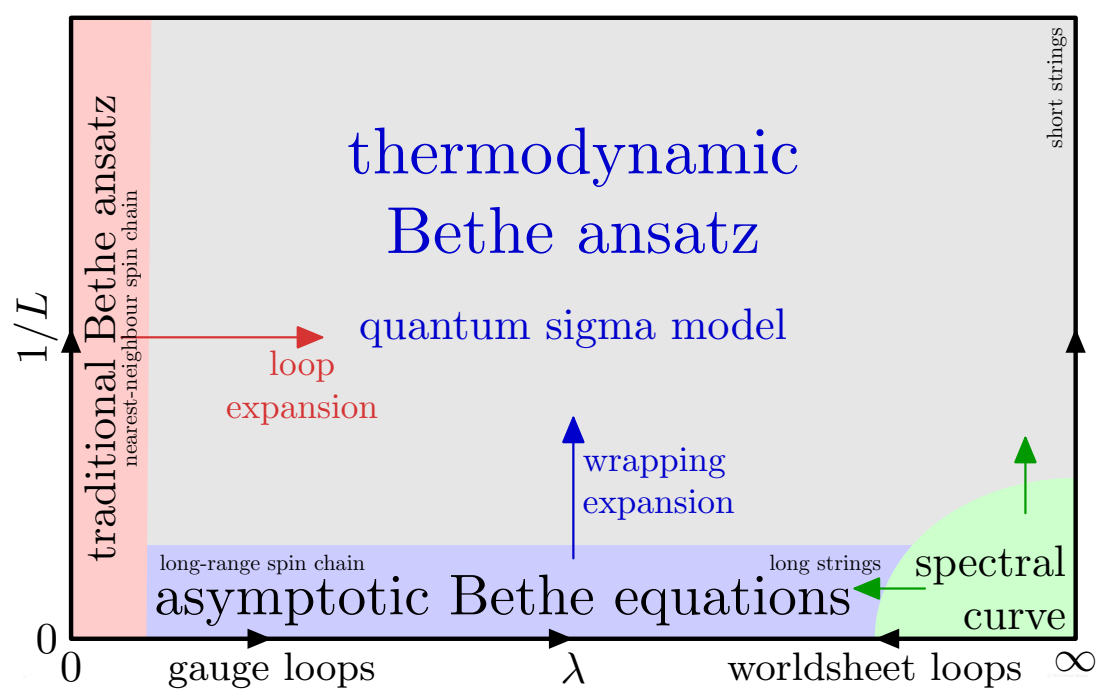

Figure 4: Phase diagram of local operators in planar $\mathcal{N}=4$ SYM mapped with respect to coupling $\lambda$ vs. "size" $L$. Also indicated are the integrability methods that describe the spectrum accurately.

and renormalisation. The final step consists in diagonalising this (potentially large) matrix. This is why scaling dimensions are solutions of algebraic equations. In comparison, the integrable approach directly predicts the algebraic equations determining the scaling dimension $D$

$$
f(D, \lambda)=0 .
$$

This is what we call a solution of the spectral problem.

A crucial benefit of integrability is that the spectral equations include the coupling constant $\lambda$ in functional form. Whereas standard methods produce an expansion whose higher loop coefficients are exponentially or even factorially hard to compute, here we can directly work at intermediate coupling strength.

What is more, integrability gives us easy access to composite objects with a large number of constituents. Generally, there is an enormous phase space for such objects growing exponentially with their size. Standard methods would require computing the complete matrix of scaling dimensions and then filter out the desired eigenvalue. Clearly this procedure is prohibitive for large sizes. Conversely, the integrable approach is formulated in terms of physically meaningful quantities. This allows us to assume a certain coherent behaviour for the constituents of the object we are interested in, and then approach the thermodynamic limit. Consequently we obtain a set of equations for the energy of just this object. Moreover, the thermodynamic limit is typically much simpler than the finite-size equations. The size of the object can be viewed as a quantum parameter, where infinite vs. finite size corresponds to classical vs. quantum physics. In fact, in many cases it does map to classical vs. quantum strings! In Fig. 4 we present a phase space for local operators in planar $\mathcal{N}=4 \mathrm{SYM}$. On it we indicate the respective integrability methods to be described in detail in the overview section and in the chapters.

As already mentioned, a strength of the integrable system approach is that objects 
are often represented through their physical parameters. This is not just an appealing feature, but also a reason for the efficiency: The framework of quantum mechanics and QFT is heavily based on equivalence classes. Explicit calculations usually work with representatives. Choosing a particular representative in a class introduces further auxiliary degrees of freedom into the system. These degrees of freedom are carried along the intermediate steps of the calculation, and it is reasonable to expect them to be a source of added difficulty because there is no physical principle to constrain their contributions. In particular, they are the habitat of the notorious infinities of QFT. At the end of the day, all of their contributions miraculously ${ }^{4}$ vanish into thin air. Hence a substantial amount of efforts typically go into calculating contributions which one is actually not interested in. Conversely, one may view integrable methods as working directly in terms of the physical equivalence classes instead of their representatives. The observables are then computed without intermediate steps or complications. The fact that such a shortcut exists for some models is a true miracle; it is called integrability.

So far we have discussed solving the spectrum of our planar model(s). A large amount of evidence has now accumulated that this is indeed possible, and, more importantly, we understand how to do it in practice. Solving the theory, however, requires much more; we should be able to compute all of its observables. For a gauge theory they include not only the spectrum of scaling dimensions, but also correlation functions, scattering amplitudes, expectation values of Wilson loops, surface operators and other extended objects, as well as combinations of these (loops with insertions, form factors, ....), if not more. For several of these, in particular for scattering amplitudes, it is becoming clear that integrability provides tools to substantially simplify their computation. Hence it is plausible to expect that the planar limit can be solved.

Can we also solve the models away from the planar limit? There are many indications that integrability breaks down for finite number of colours $N_{\mathrm{c}}$. Nevertheless, this alone does not imply that we should become dispirited. Integrability may still prove useful, not in the sense of an exact solution, but as a means to perform an expansion in terms of genus, i.e. in powers of $1 / N_{\mathrm{c}} \sim g_{\mathrm{str}}$. This might give us a new handle to approach the centre of parameter space in Fig. 2 coming from below. The centre will, with all due optimism, most likely remain a tough nut to crack.

In conclusion, methods of integrability have already brought and will continue to bring novel insights into the gauge and string models. Having many concrete results at hand helps in particular to understand their duality better. In particular we can confirm and complete the AdS/CFT dictionary which relates objects and observables between the two models.

Integrability as a Symmetry. Above we have argued that the success of integrability is based on the strict reduction to the physical degrees of freedom. Another important point of view is that integrability is a hidden symmetry. Symmetries have always been a key towards a better understanding in particle physics and QFT. Here the hidden symmetry is in fact so powerful that it not only relates selected quantities to others, but,

\footnotetext{
${ }^{4}$ Of course, the miracle is consistency of the model paired with failing to make mistakes in the calculation (often used as a convenient cross check of the result).
} 
in some sense, anything to everything else. The extended symmetry thus predicts the outcome of every measurement, at least in principle. Conventionally one would expect the resulting model to be trivial, just like a harmonic oscillator, but there are important interesting and highly non-trivial cases.

Integrability finds a natural mathematical implementation in the field of quantum algebra. More concretely, the type of quantum integrable system that we encounter is usually formulated in terms of deformed universal enveloping algebras of affine Lie algebras. The theory of such quasi-triangular Hopf algebras is in general highly developed. It provides the objects and their relations for the solution of the physical system. Curiously, our gauge/string theory integrable model appears to be based on some unconventional or exceptional superalgebra which largely remains to be understood. It is not even clear whether quasi-triangular Hopf algebras are a sufficient framework for a complete mathematical implementation of the system.

Relations to Other Subjects. An aspect which makes the topic of this review a particularly attractive one to work on is its relation to diverse subjects of theoretical physics and mathematics. Let us collect a few here, including those mentioned above, together with references to the chapters of this review where the relations are discussed in more detail:

- Most obviously, the topic of the review itself belongs to four-dimensional QFT, more specifically, gauge theory and/or CFT, but also to string theory on curved backgrounds.

- Recalling the discussion from a few lines above, the mathematical framework for the kind of integrable models that we encounter is quantum algebra, see Chapter VI.2.

- As mentioned earlier, string theory always contains a self-consistent formulation of quantum gravity. By gaining a deeper understanding of string theory models, we hope to learn more about quantum gravity as such. Furthermore, by means of the string-related Kawai-Lewellen-Tye [6] and Bern-Carrasco-Johansson relations [7], there is a connection between scattering amplitudes in $\mathcal{N}=4 \mathrm{SYM}$ and $\mathcal{N}=8$ supergravity, which stands a chance of being free of perturbative divergencies. ${ }^{5}$ These aspects are not part of the review. In fact, it would be highly desirable to explore the use of integrability results in this context.

- Prior to the discoveries related to the AdS/CFT correspondence, integrability in four-dimensional gauge theories was already observed in the context of high-energy scattering and the BFKL equations, and for deep inelastic scattering and the DGLAP equations, see Chapter IV.4 and [8]. Note that the twist states discussed in Chapters III.4 play a prominent role in deep inelastic scattering.

- There are also rather distinct applications of integrability in supersymmetric gauge theories: There is the famous Seiberg-Witten solution $[\overline{9}]$ for the BPS masses in $D=4, \mathcal{N}=2$ gauge theories. Furthermore, supersymmetric vacua in $D=2$,

\footnotetext{
${ }^{5}$ One should point out that these relations are essentially non-planar.
} 
$\mathcal{N}=4$ gauge theories with matter can be described by Bethe ansätze [10]. It remains to be seen whether there are connections to the subject of the present review.

- There are further links to general four-dimensional gauge theories: On a qualitative level we might hope to learn about QCD strings from novel results in the AdS/CFT correspondence at finite coupling. On a practical level, the leading-order results in $\mathcal{N}=4 \mathrm{SYM}$ can be carried over to general gauge theories essentially because $\mathcal{N}=4$ SYM contains all types of particles and interactions allowed in a renormalisable QFT. Chapter IV.4 is most closely related to this topic.

- Along the same lines, the BFKL dynamics in leading logarithmic approximation is universal to all four-dimensional gauge theories. The analytic expressions derived in $\mathcal{N}=4 \mathrm{SYM}$ may allow us to clarify the nature of the most interesting Regge singularity, the pomeron (see [11]), which is the most interesting object for perturbative QCD and for its applications to particle collider physics. ${ }^{6}$

- A certain class of composite states, but also loop integrals in QFT, often involve generalised harmonic sums, generalised polylogarithms and multiple zeta values. The exploration of such special functions is an active topic of mathematics. See Chapters I.2, III.4 and V.2.

- Local operators of the gauge theory are equivalent to states of a quantum spin chain. Spin chain models come to use in connection with magnetic properties in solid state physics. Also in gauge theory, ferromagnetic and anti-ferromagnetic states play an important role, see Chapters I.1 and III.4.

- More elaborate spin chains — such as the one-dimensional Hubbard model (cf. [12]) - are considered in connection to electron transport. Curiously, this rather exceptional Hubbard chain makes an appearance in the gauge theory context, in at least two distinct ways, see Chapters I.3 and III.2.

- And there are many more avenues left to be explored.

\footnotetext{
${ }^{6}$ We thank L. Lipatov for pointing out this application.
} 


\section{Outline}

The review collection consists of the above introduction and 23 chapters grouped into 6 major subjects. Each chapter reviews a particular topic in a self-contained manner. The following overview gives a brief summary of each part and each chapter, and is meant to tie the whole collection together. It can be understood as an extensive table of contents.

Where possible, we have put the chapters into a natural and meaningful order with regards to content. A chapter builds upon insights and results presented in the earlier chapters and begins roughly where the previous one ended. In many cases this reflects the historical developments, but we have tried to pull loops straight. Our aim was to prepare a pedagogical and generally accessible introduction to the subject of AdS/CFT integrability rather than a historically accurate account.

While the topics were fixed, the design and presentation of each chapter was largely the responsibility of its authors. The only guideline was to discuss an instructive example in detail while presenting the majority of results more briefly. Furthermore, the chapters give a guide to the literature relevant to the topic where more details can be found. Open problems are also discussed in the chapters. Note that we did not enforce uniform conventions for naming, use of $\mathfrak{a l p h a b e t s , ~ n o r m a l i s a t i o n s , ~ a n d ~ s o ~ o n . ~ T h i s ~ m e r e l y ~ r e f l e c t s ~}$ a reality of the literature. However, each chapter is meant to be self-consistent.

Before we begin with the overview, we would like to point out existing reviews on AdS/CFT integrability and related subjects which cover specific aspects in more detail. We can recommend several reviews dedicated to the subject [13]. Also a number of PhD theses are available which at least contain a general review as the introduction [14]. It is also worthwhile to read some of the very brief accounts of the subject in the form of news items [15]. Last but not least, we would like to refer the reader to prefaces of special issues dedicated to AdS/CFT integrability [16] and closely related subjects [17]. 


\section{Table of Contents}

page numbers refer to Lett. Math. Phys. 99 (2012), numbers $1210.0392-0405$ are arxiv.org identifiers.

\section{I $\mathcal{N}=4$ Super Yang-Mills Theory}

I.1 J. A. Minahan, "Spin Chains in $\mathcal{N}=4 S Y M$ ", pp. 33, 1012.3983.

I.2 C. Sieg, "The spectrum from perturbative gauge theory", pp. 59, 1012.3984.

I.3 A. Rej, "Long-range spin chains", pp. 85, 1012.3985.

II IIB Superstrings on $A d S_{5} \times S^{5}$

II.1 A. Tseytlin, "Classical $A d S_{5} \times S^{5}$ string solutions", pp. 103, 1012.3986.

II.2 T. McLoughlin, "Quantum Strings in $A d S_{5} \times S^{5}$ ", pp. 127, 1012.3987.

II.3 M. Magro, "Sigma Model, Gauge Fixing", pp. 149, 1012.3988.

II.4 S. Schäfer-Nameki, "The Spectral Curve", pp. 169, 1012.3989.

\section{Solving the AdS/CFT Spectrum}

III.1 M. Staudacher, "Bethe Ansätze and the R-Matrix Formalism", pp. 191, 1012.3990.

III.2 C. Ahn and R. I. Nepomechie, "Exact world-sheet S-matrix", pp. 209, 1012.3991.

III.3 P. Vieira and D. Volin, "The dressing factor", pp. 231, 1012.3992.

III.4 L. Freyhult, "Twist states and the cusp anomalous dimension", pp. 255, 1012.3993.

III.5 R. Janik, "Lüscher corrections", pp. 277, 1012.3994.

III.6 Z. Bajnok, "Thermodynamic Bethe Ansatz", pp. 299, 1012.3995.

III.7 V. Kazakov and N. Gromov, "Hirota Dynamics for Quantum Integrability", pp. 321, 1012.3996.

\section{Further Applications of Integrability}

IV.1 C. Kristjansen, "Aspects of Non-planarity", pp. 349, 1012.3997.

IV.2 K. Zoubos, "Deformations, Orbifolds and Open Boundaries", pp. 375, 1012.3998.

IV.3 T. Klose, “N $=6$ Chern-Simons and Strings on $A d S_{4} \times C P^{3}$ ", pp. 401, 1012.3999 .

IV.4 G. Korchemsky, "Integrability in $Q C D$ and $\mathcal{N}<4 S Y M$ ", pp. 425, 1012.4000.

\section{Scattering Amplitudes}

V.1 R. Roiban, "Scattering Amplitudes - a Brief Introduction", pp. 455, 1012.4001.

V.2 J. M. Drummond, "Dual Superconformal Symmetry", pp. 481, 1012.4002.

V.3 L. F. Alday, "Scattering Amplitudes at Strong Coupling", pp. 507, 1012.4003.

VI Algebraic Aspects of Integrability

VI.1 N. Beisert, "Superconformal Algebra", pp. 529, 1012.4004.

VI.2 A. Torrielli, "Yangian Algebra", pp. 547, 1012.4005. 


\section{Overview}

The chapters are grouped into six parts representing the major topics and activities of this subject, see Fig. 5. In the first two Parts I and II we start by outlining the perturbative gauge and string theory setup. Here we focus on down-to-earth quantum field theory calculations which yield the solid foundation in spectral data of local operators. In the following Part III we review the construction of the spectrum by integrable methods. More than merely reproducing the previously obtained data, this goes far beyond what could possibly be computed by conventional methods: It can apparently predict the exact spectrum. The next Part IV summarises applications of these methods to similar problems, beyond the spectrum, beyond planarity, beyond $\mathcal{N}=4 \mathrm{SYM}$ or strings on $A d S_{5} \times S^{5}$. Among these avenues is the application of integrability to scattering amplitudes; as this topic has grown into a larger subject we shall devote Part V to it. The final Part VI reviews classical and quantum algebraic aspects of the models and of integrability.



Figure 5: Suggested order of study. 


\section{I $\mathcal{N}=4$ Super Yang-Mills Theory}

This part deals with the maximally supersymmetric Yang-Mills $(\mathcal{N}=$ 4 SYM) theory in four spacetime dimensions. This model is a straightforward quantum field theory. It uses the same types of particles and interactions that come to play in the Standard Model of particle physics. However, the particle spectrum and the interactions are


delicately balanced granting the model a host of unusual and unexpected features. The best-known of these is exact (super)conformal symmetry at the quantum level. A far less apparent feature is what this review collection is all about: integrability.

In this part we focus on the perturbative field theory, typically expressed through Feynman diagrams. The calculations are honest and reliable but they become tough as soon as one goes to higher loop orders. Integrability will only be discussed as far as it directly concerns the gauge theory setup, i.e. in the sense of conserved operators acting on a spin chain. The full power of integrability will show up only in Part III.

\section{I.1 Spin Chains in $\mathcal{N}=4$ SYM}

Chapter I.1 introduces the gauge theory, its local operators, and outlines how to compute the spectrum of their planar one-loop anomalous dimensions. It is explained how to map one-to-one local operators to states of a certain quantum spin chain. The operator which measures

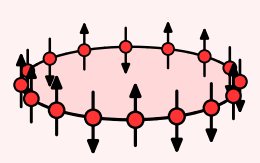
the planar, one-loop anomalous dimensions corresponds to the spin chain Hamiltonian in this picture. Importantly, this Hamiltonian is of the integrable kind, and the planar model can be viewed as a generalisation of the Heisenberg spin chain. This implies that its spectrum is solved efficiently by the corresponding Bethe ansatz. E.g. a set of one-loop planar anomalous dimensions $\delta D$ is encoded in the solutions of the following set of Bethe equations for the variables $u_{k} \in \mathbb{C}(k=1, \ldots, M)$

$$
\left(\frac{u_{k}+\frac{i}{2}}{u_{k}-\frac{i}{2}}\right)^{L}=\prod_{\substack{j=1 \\ j \neq k}}^{M} \frac{u_{k}-u_{j}+i}{u_{k}-u_{j}-i}, \quad 1=\prod_{j=1}^{M} \frac{u_{j}+\frac{i}{2}}{u_{j}-\frac{i}{2}}, \quad \delta D=\frac{\lambda}{8 \pi^{2}} \sum_{j=1}^{M} \frac{1}{u_{j}^{2}+\frac{1}{4}} .
$$

Finally the Chapter presents applications of the Bethe ansatz to sample problems. 


\section{I.2 The spectrum from perturbative gauge theory}

The following Chapter I.2 reviews the computation of the spectrum of anomalous dimensions at higher loops in perturbative gauge theory. The calculation in terms of Feynman diagrams is firmly established, but just a handful orders takes you to the limit of what is generally possible. Computer algebra and superspace techniques push the limit

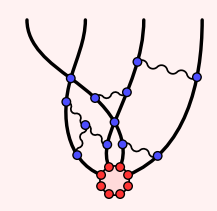
by a few orders.

In our case the results provide a valuable set of irrefutable data which the integrable model approach must be able to reproduce to show its viability. This comprises not only explicit energy eigenvalues, but also crucial data for the integrable system, such as the magnon dispersion relation and scattering matrix. Importantly, also the leading finite-size terms are accessible in this approach, e.g. the four-loop anomalous dimension to the simplest non-trivial local operator reads (cf. the above Bethe equation with $L=$ $4, M=2)$

$$
\delta D=\frac{3 \lambda}{4 \pi^{2}}-\frac{3 \lambda^{2}}{16 \pi^{4}}+\frac{21 \lambda^{3}}{256 \pi^{6}}-\frac{(78-18 \zeta(3)+45 \zeta(5)) \lambda^{4}}{2048 \pi^{8}}+\ldots
$$

\section{I.3 Long-range spin chains}

The final Chapter I.3 of this part reviews spin chain Hamiltonians originating in planar gauge theory at higher loops. The one-loop Hamiltonian describes interactions between two neighbouring spins. At higher loops the Hamiltonian is deformed by interactions between several neighbouring spins, e.g.

$$
H=\sum_{j=1}^{L}\left(\frac{\lambda}{8 \pi^{2}}\left(1-P_{j, j+1}\right)+\frac{\lambda^{2}}{128 \pi^{4}}\left(-3+4 P_{j, j+1}-P_{j, j+2}\right)+\ldots\right) .
$$

Moreover, the Hamiltonian can dynamically add or remove spin sites! While integrable nearest-neighbour Hamiltonians have been studied in detail for a long time, a better general understanding of long-range deformations was developed only recently. Curiously, several well-known integrable spin chain models make an appearance in this context, in particular, the Haldane-Shastry, Inozemtsev and Hubbard models. 


\section{IIB Superstrings on $A d S_{5} \times S^{5}$}

This part concerns IIB string theory on the maximally supersymmetric $A d S_{5} \times S^{5}$ background. The string worldsheet model is a twodimensional UV-finite quantum field theory. It is of the non-linear sigma model kind with target space $A d S_{5} \times S^{5}$ and further possesses worldsheet diffeomorphisms. Also this model has a number of ex-

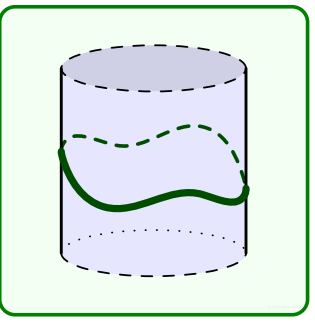
ceptional features, such as kappa symmetry, which make it a viable string theory on a stable background. Somewhat less surprising than in gauge theory, this model is also integrable, a property shared by many two-dimensional sigma models on coset spaces.

We outline how to extract spectral data from classical string solutions with quantum corrections. There are many complications, such as non-linearity of the classical equations of motion, lack of manifest supersymmetry and presence of constraints. Again, integrability will help tremendously; here we focus on string-specific aspects, and leave the more general applications to Part III.

\section{II.1 Classical $A d S_{5} \times S^{5}$ string solutions}

The first Chapter II.1 of this part introduces the Green-Schwarz string on the curved spacetime $A d S_{5} \times S^{5}$. For the classical spectrum only the bosonic fields are relevant. To find exact solutions of the non-linear equations of motions, one typically makes an ansatz for the shape of the string. Taking inspiration from spinning strings in flat space, one


can for instance assume a geodesic rod spinning around some orthogonal axes. The equations of motion together with the Virasoro constraints dictate the local evolution, while boundary conditions quantise the string modes. Next, the target space isometries give rise to conserved charges, such as angular momenta and energy. These can be expressed in terms of the parameters of the string solution. E.g., a particular class of spinning strings on $A d S_{3} \times S^{1} \subset A d S_{5} \times S^{5}$ obeys the following relation (K, E are elliptic integrals)

$$
\frac{S^{2}}{(\mathrm{~K}(m)-\mathrm{E}(m))^{2}}-\frac{J^{2}}{\mathrm{~K}(m)^{2}}=16 n^{2} T^{2}(1-m), \quad \frac{J^{2}}{\mathrm{~K}(m)^{2}}-\frac{E^{2}}{\mathrm{E}(m)^{2}}=16 n^{2} T^{2} m .
$$

Such relations can be used to express the energy $E$ as a function of the angular momenta $J, S$, the string modes $n$ and the string tension $T .^{7}$

\footnotetext{
${ }^{7}$ Note that complicated classes of solutions will require further internal parameters in addition to $m$.
} 


\section{II.2 Quantum Strings in $A d S_{5} \times S^{5}$}

Chapter II.2 continues with semiclassical quantisation of strings. Here, one distinguishes between point-like and extended strings.

Quantisation around point-like strings is the direct analogue of what is commonly done in flat space. The various modes of the string can be excited in quantised amounts, and the string spectrum takes the form

$$
E-J=\sum_{k=1}^{M} N_{k} \sqrt{1+\lambda n_{k}^{2} / J^{2}}+\ldots, \quad \sum_{k=1}^{M} N_{k} n_{k}=0 .
$$

The main difference with flat space is that the modes interact, adding non-trivial corrections to the spectrum. These corrections can be computed in terms of a scattering problem on the worldsheet.

Quantisation around extended string solutions is far less trivial: The spectrum of fluctuations now crucially depends on the classical solution. Another effect is that the energy of the classical string receives quantum corrections due to vacuum energies of the string modes.

\section{II.3 Sigma Model, Gauge Fixing}

Spheres and anti-de-Sitter spacetimes are symmetric cosets. Chapter II.3 presents the formulation of the string worldsheet as a twodimensional coset space sigma model of the target space isometry supergroup. In particular, integrability finds a simple formulation in a family of flat connections $A(z)$ on the worldsheet and its holonomy

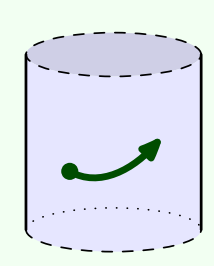
$M(z)$ around the closed worldsheet

$$
d A(z)+A(z) \wedge A(z)=0, \quad M(z)=\mathrm{P} \exp \oint A(z)
$$

Series expansion of $M(z)$ in the spectral parameter $z$ leads to an infinite tower of charges extending the isometries to an infinite-dimensional algebra.

Proper treatment of symmetries and integrability towards a canonical quantisation requires a Hamiltonian formulation. Here the major complications are the presence of first and second class constraints due to worldsheet diffeomorphisms and kappa symmetry. Finally, one encounters notorious ambiguities in deriving the algebra of conserved charges. 


\section{II.4 The Spectral Curve}

In the final Chapter II.4 on strings, the flat connection is applied to the construction of the (semi)classical string spectrum. The eigenvalues $e^{i p_{k}(z)}$ of the monodromy $M(z)$ are integrals of motion. As functions of complex $z$ they define a spectral curve for each classical solution.

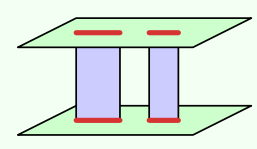
Instead of studying explicit classical solutions we can now study abstract spectral curves. Besides containing all the spectral information, they offer a neat physical picture: String modes correspond to handles of the Riemann surface, and each handle has two associated moduli: the mode number $n_{k}$ and an amplitude $\alpha_{k}$. They can be extracted easily as periods of the curve

$$
\oint_{A_{k}} d p=0, \quad \frac{1}{2 \pi} \oint_{B_{k}} d p=n_{k}, \quad \frac{\sqrt{\lambda}}{4 \pi^{2} i} \oint_{A_{k}} \frac{1+z^{4}}{1-z^{4}} d p=N_{k} .
$$

Note that quantisation replaces the amplitude by an integer excitation number $N_{k}$ thus providing access to the semiclassical spectrum of fluctuation modes.

\section{Solving the AdS/CFT Spectrum}

Armed with some basic knowledge of the relevant structures in gauge and string theory (as well as an unconditional belief in the applicability of integrable structures to this problem) we aim to solve the planar spectrum in this part.

The starting point is that in both models there is a one-

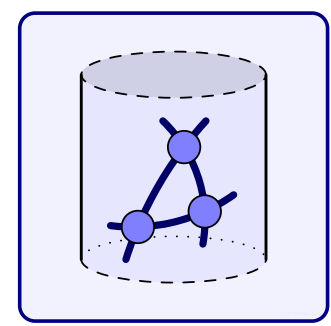
dimensional space (spin chain, string) on which some particles (magnons, excitations) can propagate. By virtue of symmetry and integrability one can derive how they scatter, at all couplings and in all directions. Taking periodicity into account properly, one arrives at a complete and exact description of the spectrum. For certain states this program was carried out, and all results are in complete agreement with explicit calculations in perturbative gauge or string theory (as far as they are available). Yet, the results from the integrable system approach go far beyond what is otherwise possible in QFT: They provide a window to finite coupling $\lambda$ !

There are several proposals of how to formulate these equations - through an algebraic system or through integral equations. However, it is commonly believed that a reasonably simple and generally usable form for such equations has not yet been found (let alone proved). 


\section{III.1 Bethe Ansätze and the R-Matrix Formalism}

As a warm-up exercise and to gather experience, Chapter III.1 solves one of the oldest quantum mechanical systems - the Heisenberg spin chain. This is done along the lines of Bethe's original work, using a factorised magnon scattering picture, but also in several variants of the Bethe ansatz. This introduces us to ubiquitous concepts of integrable

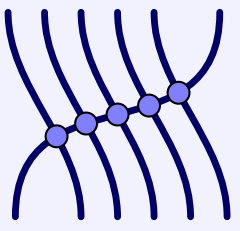
systems such as R-matrices, transfer matrices and the famous Yang-Baxter equation

$$
R_{12} R_{13} R_{23}=R_{23} R_{13} R_{12}
$$

The chapter ends by sketching a promising novel method for constructing the so-called Baxter Q-operators, allowing to surpass the Bethe ansatz technique.

\section{III.2 Exact world-sheet S-matrix}

Even though little is known about gauge or string theory at finite coupling, the magnon scattering pictures and symmetries qualitatively agree for weak and strong coupling. Under the assumption that they remain valid at intermediate couplings, Chapter III.2 describes how to make use of symmetry to determine all the relevant quantities: Both,


the magnon dispersion relation

$$
e(p)=\sqrt{1+\frac{\lambda}{\pi^{2}} \sin ^{2}\left(\frac{1}{2} p\right)}
$$

and the 16-flavour scattering matrix are almost completely determined through representation theory of an extended $\operatorname{psu}(2 \mid 2)$ superalgebra. Integrability then ensures factorised scattering, and determines the spectrum on sufficiently long chains or strings through the asymptotic Bethe equations.

\section{III.3 The dressing factor}

Symmetry alone cannot predict an overall phase factor of the scattering matrix which is nevertheless crucial for the spectrum. Several other desirable properties of factorised scattering systems, such as unitarity, crossing and fusion, constrain its form

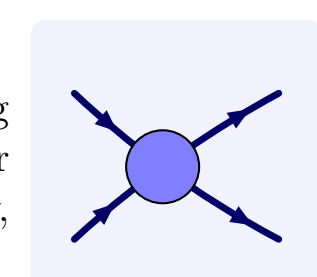

$$
S_{12}^{0} S_{1 \overline{2}}^{0}=f_{12} .
$$

Chapter III.3 presents this crossing equation and its solution - the so-called dressing phase. It has a host of interesting analytic properties relating to the physics of the model under discussion. 


\section{III.4 Twist states and the cusp anomalous di- mension}

The asymptotic Bethe equations predict the spectrum up to finite-size corrections. In Chapter III.4 we apply them to the interesting class of twist states. These are ideally suited for testing purposes because a lot of solid spectral data are known from perturbative gauge and string theory. They also have an interesting dependence on their spin $j$, in terms of generalised harmonic sums of fixed degree.

Importantly, in the large spin limit, finite-size corrections turn out to be suppressed. The Bethe equations reduce to an integral equation to predict the exact cusp dimension (and generalisations). The latter turns out to interpolate smoothly between weak and strong coupling in full agreement with perturbative data

$$
D_{\text {cusp }}=\frac{\lambda}{2 \pi^{2}}-\frac{\lambda^{2}}{96 \pi^{2}}+\frac{11 \lambda^{3}}{23040 \pi^{2}}+\ldots=\frac{\sqrt{\lambda}}{\pi}-\frac{3 \log 2}{\pi}-\frac{\beta(2)}{\pi \sqrt{\lambda}}+\ldots
$$

\section{III.5 Lüscher corrections}

For generic states, however, finite-size corrections are required to get agreement with gauge and string theory. Chapter III.5 explains how to apply Lüscher terms to determine these: On a closed worldsheet there are virtual particles propagating in the spatial direction in non-trivial loops around the string. When they interact with physical excitations,


they give rise to non-trivial energy shifts $\left(q_{j}\right.$ and $p_{k}$ are virtual and real particle momenta, respectively)

$$
\delta E=-\sum_{j} \int \frac{d q_{j}}{2 \pi} e^{-L \tilde{e_{j}}\left(q_{j}\right)} \operatorname{STr}_{j} \prod_{k} S_{j k}\left(q_{j}, p_{k}\right)
$$

\section{III.6 Thermodynamic Bethe Ansatz}

Although finite-size corrections appear under control, it is clearly desirable to find equations to determine the exact spectrum in one go. Chapter III.6 describes the thermodynamic Bethe ansatz approach based on the following idea: Consider the string worldsheet at finite temperature with Wick rotated time. It has the topology of a torus of

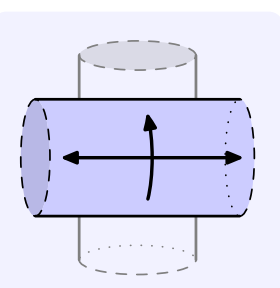
radius $R$ and time period $L$. We are primarily interested in the zero temperature limit where time is decompactified. Now the torus partition function can be evaluated in the mirror theory where the periods are exchanged

$$
Z(R, L)=\tilde{Z}(L, R)
$$

Then, instead of time, we can decompactify the radius. Conveniently, the asymptotic Bethe equations become exact, and eventually predict the finite-size spectrum. 


\section{III.7 Hirota Dynamics for Quantum Integrability}

Chapter III.7 presents a equivalent proposal for the finite-size spectrum based on the conserved charges of an integrable model. The latter are typically packaged into transfer matrix eigenvalues $T(u)$. These exist in various instances which obey intricate relations, such as the discrete Hirota equation (also known as the Y-system for equivalent quantities $\left.Y_{a, s}(u)\right)$

$$
T_{a, s}\left(u+\frac{i}{2}\right) T_{a, s}\left(u-\frac{i}{2}\right)=T_{a+1, s}(u) T_{a-1, s}(u)+T_{a, s+1}(u) T_{a, s-1}(u) .
$$

Similarly to Chapter II.4, one can start from these equations, subject to suitable boundary conditions, and predict the spectrum at finite coupling.

\section{Further Applications of Integrability}

For the sake of a clear presentation the previous parts focused on one particular application of integrability in AdS/CFT: Solving the exact planar spectrum of $\mathcal{N}=4$ supersymmetric Yang-Mills theory or equivalently of IIB string theory on $A d S_{5} \times S^{5}$. While this topic has been at the centre of attention, many investigations have dealt


with extending the applications of integrability to other observables beyond the planar spectrum and to more general models. This part and the following try to give an overview of these developments.

\section{IV.1 Aspects of Non-planarity}

Integrability predicts the planar spectrum accurately and with minimum effort. It would be desirable to extend the applications of integrability to non-planar corrections because, e.g., in QCD $N_{\mathrm{c}}=3$ rather than $N_{\mathrm{c}}=\infty$. For the spectrum, these are interactions where the spin chain or the string splits up and recombines

$$
H=H_{0}+\frac{1}{N_{\mathrm{c}}}\left(H_{+}+H_{-}\right)+\ldots
$$

They result in a string worldsheet of higher genus or with more than two punctures.

Chapter IV.1 reviews the available results on higher-genus corrections, higher-point functions as well as supersymmetric Wilson loops in the AdS/CFT context. It is shown that most of the basic constructions of integrability do not work in the non-planar setup. 


\section{IV.2 Deformations, Orbifolds and Open Bound- aries}

There exist many deformations of $\mathcal{N}=4 \mathrm{SYM}$ which preserve some or the other property, e.g. by deforming the $(\mathcal{N}=1)$ superpotential

$$
\int d^{4} x d^{4} \theta \operatorname{Tr}\left(e^{i \beta} X Y Z-e^{-i \beta} Z Y X\right)
$$

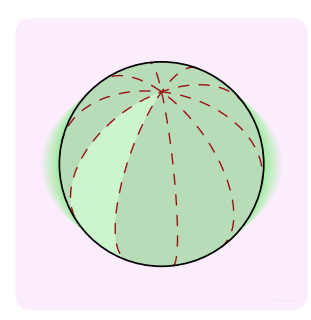

It is natural to find out under which conditions integrability can survive. Chapter IV.2 reviews such superconformal deformations of $\mathcal{N}=4 \mathrm{SYM}$ and shows how the methods of integrability can be adjusted to these cases. It turns out that these merely deform the boundary conditions of the integrable model by introducing additional phases into the Bethe equations (in the spin chain context this has a similar effect as turning on a magnetic field). Different boundary conditions can also arise from looking at other corners of the spectrum or at different observables; this is another topic of the present chapter.

\section{IV.3 $\mathcal{N}=6$ Chern-Simons and Strings on $\mathrm{AdS}_{4} \times \mathrm{CP}^{3}$}

Recently a quantum field theory in three dimensions was discovered which behaves in many respects like $\mathcal{N}=4 \mathrm{SYM}-\mathcal{N}=6$ supersymmetric Chern-Simons-matter theory

$$
S=\frac{k}{4 \pi} \int \operatorname{Tr}\left(A \wedge d A+\frac{2}{3} A \wedge A \wedge A+\ldots\right) .
$$

It is exactly superconformal at the quantum level, and there is an AdS/CFT dual string theory - IIA superstrings on the $A d S_{4} \times C P^{3}$ background. Importantly, there exists a large- $N_{\mathrm{c}}$ limit, in which the model becomes integrable. Chapter IV.3 reviews integrability in this $\mathrm{AdS}_{4} / \mathrm{CFT}_{3}$ correspondence. While being largely analogous to the constructions in the previous parts, there are several noteworthy differences in the application of integrable methods: For instance, here the spin representation alternates between the sites.

\section{IV.4 Integrability in QCD and $\mathcal{N}<4$ SYM}

Similar integrable structures were known to exist in more general gauge theories long before the exploitation of integrability in $\mathcal{N}=4$ SYM. Chapter IV.4 introduces evolution equations for high-energy scattering (BFKL) and scaling of quasi-partonic operators in connection to deep inelastic scattering (DGLAP). To some extent these take the form of integrable Hamiltonians with $\operatorname{sl}(2 \mid \mathcal{N})$ symmetry $\left(J_{12}\right.$ is the two-particle spin operator and $\Psi$ is the digamma function)

$$
H_{12} \simeq \Psi\left(J_{12}\right)-\Psi(1)
$$

Its eigenvalues determine the scaling of certain hadronic structure functions and control the energy dependence of scattering amplitudes in the high-energy (Regge) limit. 


\section{Integrability for Scattering Amplitudes}

The most conservative application of quantum field theories is to compute scattering cross sections (to be compared to particle scattering experiments). With old blades sharpened and new ones developed, the charted territory of tree and loop scattering amplitudes in $\mathcal{N}=4$ SYM has increased dramatically, see e.g. the recent reviews [18] and

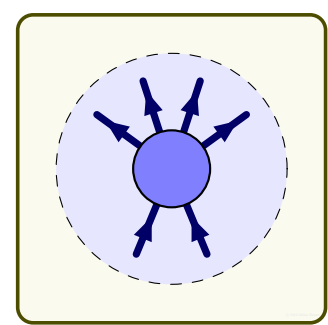
the special issue [19]. It was soon noticed that something special was going on in the planar limit which makes amplitudes much simpler than originally thought. It does not take much imagination to conjecture a connection to integrability. This part reviews scattering amplitudes and what integrability implies in this context. This topic is under active investigation, many advances have been and are being made, but a lot remains to be understood. Here, one can expect that integrability will enable a similarly simple solution as in the case of the planar spectrum.

\section{V.1 Scattering Amplitudes - a Brief Introduction}

Chapter V.1 gives an introduction into the topic of scattering amplitudes in $\mathcal{N}=4 \mathrm{SYM}$. First of all, the spinor-helicity formalism and colour-ordering scheme strips the combinatorial structure and leaves plain functions. For instance, an essential part of an $n$-particle amplitude simply reads $(\langle k j\rangle$ is a Lorentz-invariant constructed from the


momenta of particles $k$ and $j$ )

$$
A_{n}^{\mathrm{MHV}}=\frac{\delta^{4}(P) \delta^{8}(Q)}{\langle 12\rangle\langle 23\rangle \cdots\langle n 1\rangle} .
$$

The S-matrix displays a host of useful analyticity properties related to unitarity. These can be used to reconstruct tree and loop amplitudes from scratch, which is typically far more efficient than using Feynman diagrams following from the Lagrangian description.

\section{V.2 Dual Superconformal Symmetry}

Chapter V.2 reviews simplifications found in planar scattering. It turns out that the underlying scalar integrals are of a special form which hints at conformal symmetry in a dual space. Indeed, the amplitudes obey a dual superconformal symmetry in addition to the conventional one. The two sets of conformal symmetries close onto an infinite-dimensional


algebra which is at the heart of integrability — the Yangian.

This symmetry helps to determine all (tree) amplitudes, by means of recursion or through a Grassmannian integral ( $C$ is a $k \times n$ matrix, $M_{j}$ are its $k \times k$ minors of consecutive columns, and $Z$ are $4 \mid 4$ twistors encoding the momenta of the $n$ legs)

$$
A_{n, k}^{\mathrm{tree}}(Z)=\int \frac{d^{k(n-k)} C \delta^{k(4 \mid 4)}(C Z)}{M_{1} \cdots M_{n}}
$$




\section{V.3 Scattering Amplitudes at Strong Coupling}

Chapter V.3 discusses the string dual of scattering amplitudes. Here it makes sense to transform particle momenta to distances by means of a T-duality. At strong coupling an amplitude is then dominated by the minimal area of a string worldsheet ending on a light-like polygonal contour on the $A d S_{5}$ boundary (the previous Chapter V.2 provides ev-

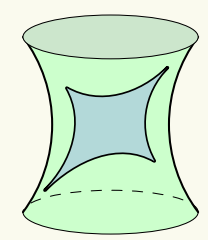
idence in favour of a general relation between amplitudes and light-like polygonal Wilson loops). Such minimal areas can be computed efficiently by integrable means bypassing the determination of the complicated shape of the worldsheet (cf. Chapter III.5)

$$
A_{\text {reg }}=\sum_{k} \int \frac{d \theta m_{k} \cosh \theta}{2 \pi} \log \left(1+Y_{k}(\theta)\right) .
$$

\section{Algebraic Aspects of Integrability}

Integrability can be viewed as a symmetry. In most cases it enhances an obvious, finite-dimensional symmetry of a physical system to a hidden, infinite-dimensional algebra. The extended symmetry then imposes a large number of constraints onto the system which determine the dynamics (almost) completely, but without making it trivial.

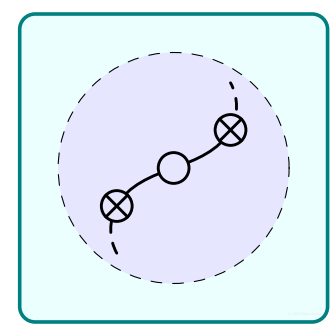
Many of the properties and methods that come to use in integrable systems find a mathematical formulation in terms of quantum algebra. Often this does not help immediately in computing particular physical observables, one of the main objectives of the previous parts. Rather, it can give a deeper understanding of how the model's integrability works, with a view to finding rigorous proofs for the applicability of the (well-tested) proposals.

This final part of the review presents the symmetries relevant to our gauge and string theory problem. These are the Lie supergroup $\operatorname{PSU}(2,2 \mid 4)$ as the obvious symmetry and its Yangian algebra to encode integrability.

\section{VI.1 Superconformal Algebra}

The Lie superalgebra $\operatorname{psu}(2,2 \mid 4)$ is generated by $8 \times 8$ supermatrices (in $2|4| 2$ grading)

$$
J=\left(\begin{array}{c|c|c}
L & -i Q & P \\
\hline S & R & \bar{Q} \\
\hline K & -i \bar{S} & \bar{L}
\end{array}\right),
$$

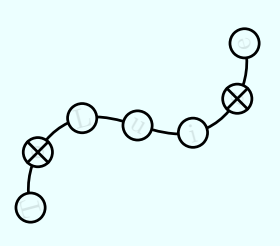

subject to suitable constraints, projections and hermiticity conditions. It serves as the spacetime superconformal symmetry in gauge theory as well as the target space isometries of the dual string theory.

Chapter VI.1 summarises some well-known facts and results for this algebra. It also explains how the algebra applies to the gauge and string theory setup. The chapter is not so much related to integrability itself, it can rather be understood as an appendix to many of the other chapters when it comes to the basics of symmetry. 


\section{VI.2 Yangian Algebra}

In physics one is used to the concept of locally and homogeneously acting symmetries. Chapter VI.2 introduces the Yangian algebra whose non-local action is encoded by the coproduct

$$
\Delta\left(Y^{A}\right)=Y^{A} \otimes 1+1 \otimes Y^{A}+f_{B C}^{A} J^{B} \otimes J^{C} .
$$

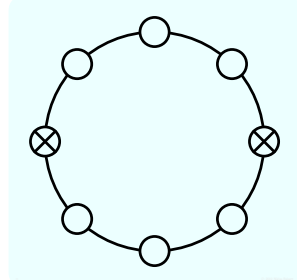

For instance, such a non-local action permits a scattering matrix which is fully determined by the algebra while still being non-trivial. The scattering matrix becomes a natural intertwining object of the Yangian, its R-matrix. It enjoys a host of useful properties which eventually make the physical system tractable.

\section{Acknowledgements}

N. Beisert thanks T. Bargheer, M. de Leeuw and L. Lipatov for helpful comments on the manuscript.

The work of C. Ahn was supported in part by KRF-2007-313-C00150 and WCU grant R32-2008-000-10130-0 (CA). R. Janik was supported in part by Polish science funds as a research project N N202 105136 (2009-2011). The work of C. Kristjansen and K. Zoubos was supported by FNU through grant number 272-08-0329. The work of R. I. Nepomechie was supported in part by the National Science Foundation under Grants PHY-0554821 and PHY-0854366 (RN). The work of A. Torrielli has been partially supported by EPSRC through the grant no. EP/H000054/1.

\section{References}

[1] J. M. Maldacena, "The large $N$ limit of superconformal field theories and supergravity", Adv. Theor. Math. Phys. 2, 231 (1998), hep-th/9711200. • S. S. Gubser, I. R. Klebanov and A. M. Polyakov, "Gauge theory correlators from non-critical string theory", Phys. Lett. B428, 105 (1998), hep-th/9802109. • E. Witten, "Anti-de Sitter space and holography", Adv. Theor. Math. Phys. 2, 253 (1998), hep-th/9802150.

[2] S. Kovacs, "N=4 supersymmetric Yang-Mills theory and the AdS/SCFT correspondence", hep-th/9908171. • O. Aharony, S. S. Gubser, J. M. Maldacena, H. Ooguri and Y. Oz, "Large N field theories, string theory and gravity", Phys. Rept. 323, 183 (2000), hep-th/9905111. • E. D'Hoker and D. Z. Freedman, "Supersymmetric gauge theories and the AdS/CFT correspondence", hep-th/0201253. J. M. Maldacena, "Lectures on AdS/CFT", hep-th/0309246. • H. Nastase, "Introduction to AdS-CFT", arxiv:0712.0689. - J. Polchinski, "Introduction to Gauge/Gravity Duality", arxiv:1010.6134.

[3] M. K. Benna and I. R. Klebanov, "Gauge-String Dualities and Some Applications", arxiv:0803.1315. • I. R. Klebanov and J. M. Maldacena, "Solving quantum field theories via curved spacetimes", Phys. Today 62, 28 (2009).

[4] G. 't Hooft, "A Planar Diagram Theory for Strong Interactions", Nucl. Phys. B72, 461 (1974) 
[5] G. T. Horowitz and J. Polchinski, "Gauge / gravity duality", gr-qc/0602037

[6] H. Kawai, D. C. Lewellen and S.-H. H. Tye, "A relation between tree amplitudes of closed and open strings", Nucl. Phys. B269, 1 (1986).

[7] Z. Bern, J. J. M. Carrasco and H. Johansson, "New Relations for Gauge-Theory Amplitudes", Phys. Rev. D78, 085011 (2008), arxiv:0805.3993. • Z. Bern, J. J. M. Carrasco and H. Johansson, "Perturbative Quantum Gravity as a Double Copy of Gauge Theory", Phys. Rev. Lett. 105, 061602 (2010), arxiv: 1004.0476.

[8] B. L. Ioffe, V. S. Fadin and L. N. Lipatov, (eds.), "Quantum chromodynamics: Perturbative and nonperturbative aspects", Cambridge University Press (2010), Cambridge, UK.

[9] N. Seiberg and E. Witten, "Monopole Condensation, And Confinement In $\mathcal{N}=2$ Supersymmetric Yang-Mills Theory", Nucl. Phys. B426, 19 (1994), hep-th/9407087. N. Seiberg and E. Witten, "Monopoles, duality and chiral symmetry breaking in $\mathcal{N}=2$ supersymmetric QCD", Nucl. Phys. B431, 484 (1994), hep-th/9408099.

[10] N. A. Nekrasov and S. L. Shatashvili, "Supersymmetric vacua and Bethe ansatz", Nucl. Phys. Proc. Suppl. 192-193, 91 (2009), arxiv:0901.4744. • N. A. Nekrasov and S. L. Shatashvili, "Quantum integrability and supersymmetric vacua", Prog. Theor. Phys. Suppl. 177, 105 (2009), arxiv:0901.4748.

[11] H. Lotter, "Phenomenology of the BFKL pomeron and unitarity corrections at low $x$ ", hep-ph/9705288. - J. R. Forshaw and D. A. Ross, "Quantum chromodynamics and the pomeron", Cambridge University Press (1997), Cambridge, UK. • S. Donnachie, G. Dosch, P. Landshoff and O. Nachtmann, "Pomeron Physics and QCD", Cambridge University Press (2002), Cambridge, UK.

[12] F. H. L. Essler, H. Frahm, F. Göhmann, A. Klümper and V. E. Korepin, "The one-dimensional Hubbard model", Cambridge University Press (2005), Cambridge, UK.

[13] J. Plefka, "Spinning strings and integrable spin chains in the AdS/CFT correspondence", Living Rev. Rel. 8, 9 (2005), hep-th/0507136. - J. A. Minahan, "A brief introduction to the Bethe ansatz in $\mathcal{N}=4$ super-Yang-Mills", J. Phys. A39, 12657 (2006). • N. Dorey, "Notes on integrability in gauge theory and string theory", J. Phys. A42, 254001 (2009). G. Arutyunov and S. Frolov, "Foundations of the $A d S_{5} \times S^{5}$ Superstring. Part I", J. Phys. A42, 254003 (2009), arxiv:0901.4937. • B. Basso and G. P. Korchemsky, "Nonperturbative scales in AdS/CFT", J. Phys. A42, 254005 (2009), arxiv:0901.4945. L. F. Alday, "Scattering amplitudes and the AdS/CFT correspondence", J. Phys. A42, 254006 (2009). • D. Serban, "Integrability and the AdS/CFT correspondence", J. Phys. A44, 124001 (2011), arxiv:1003.4214. • F. Fiamberti, A. Santambrogio and C. Sieg, "Superspace methods for the computation of wrapping effects in the standard and beta-deformed $\mathcal{N}=4 S Y M "$, arxiv:1006.3475.

[14] N. Beisert, "The dilatation operator of $\mathcal{N}=4$ super Yang-Mills theory and integrability", Phys. Rept. 405, 1 (2005), hep-th/0407277. • I. Swanson, "A review of integrable deformations in AdS/CFT", Mod. Phys. Lett. A22, 915 (2007), arxiv:0705.2844. K. Okamura, "Aspects of Integrability in AdS/CFT Duality", arxiv:0803.3999. B. Vicedo, "Finite-g Strings", J. Phys. A 44, 124002 (2011), arxiv:0810.3402. • A. Rej, "Integrability and the AdS/CFT correspondence", J. Phys. A42, 254002 (2009), arxiv:0907.3468. - N. Gromov, "Integrability in AdS/CFT correspondence: 
Quasi-classical analysis", J. Phys. A42, 254004 (2009). • D. Volin, "Quantum integrability and functional equations", J. Phys. A44, 124003 (2011), arxiv:1003.4725. V. G. M. Puletti, "On string integrability. A journey through the two-dimensional hidden symmetries in the AdS/CFT dualities", Adv. High Energy Phys. 2010, 471238 (2010), arxiv:1006.3494. - M. de Leeuw, "The S-matrix of the $A d S_{5} \times S^{5}$ superstring", arxiv: 1007.4931

[15] S. Schäfer-Nameki, "Strings and super-Yang-Mills theory: The integrable story", J. Stat. Mech. 0612, N001 (2006) • H. Nicolai, "String theory: Back to basics", Nature 449, 797 (2007).

[16] C. Kristjansen, M. Staudacher and A. Tseytlin, (eds.), "Gauge-string duality and integrability: Progress and outlook", J. Phys. A42, 250301 (2009). • P. Dorey, J. Minahan and A. Tseytlin, (eds.), "Quantum integrable models and gauge-string duality", J. Phys. A44, 120301 (2011)

[17] P. Dorey, G. Dunne and J. Feinberg, (eds.), "Recent Advances in Low-Dimensional Quantum Field Theories", J. Phys. A39, issue 41 editorial (2006). • F. Alcaraz, O. Babelon, J. de Gier and O. Foda, (eds.), "The 75th Anniversary of the Bethe Ansatz", topical articles in J. Stat. Mech., http://iopscience.iop.org/1742-5468/focus/extra.topical2

[18] L. J. Dixon, "Gluon scattering in $\mathcal{N}=4$ super-Yang-Mills theory from weak to strong coupling", PoS RADCOR2007, 056 (2007), arxiv:0803.2475. • L. F. Alday and R. Roiban, "Scattering Amplitudes, Wilson Loops and the String/Gauge Theory Correspondence", Phys. Rept. 468, 153 (2008), arxiv:0807.1889. • J. M. Henn, "Duality between Wilson loops and gluon amplitudes", Fortsch. Phys. 57, 729 (2009),

arxiv:0903.0522. • M. Wolf, "A First Course on Twistors, Integrability and Gluon Scattering Amplitudes", J. Phys. A43, 393001 (2010), arxiv:1001.3871.

J. M. Drummond, "Hidden Simplicity of Gauge Theory Amplitudes", Class. Quant. Grav. 27, 214001 (2010), arxiv:1010.2418.

[19] R. Roiban, M. Spradlin and A. Volovich, (eds.), "Scattering Amplitudes in Gauge Theories: Progress and Outlook", J. Phys. A 44, 450301 (2011). 